Full citation:

V. Mozgovoy, "Stress Pattern Recognition Through Wearable Biosensors in the Workplace: Experimental Longitudinal Study on the Role of Motion Intensity," 2019 6th Swiss Conference on Data Science (SDS), Bern, Switzerland, 2019, pp. 37-45.

doi:10.1109/SDS.2019.00-10

Link to the final, published article in IEEE Xplore:

http://ieeexplore.ieee.org/stamp/stamp.jsp?tp=\&arnumber=8789858\&isnumber=8789845

Copyright (C) 2019 IEEE. by The Institute of Electrical and Electronics Engineers, Inc. All rights reserved. 


\title{
Stress Pattern Recognition Through Wearable Biosensors in the Workplace: Experimental Longitudinal Study on the Role of Motion Intensity
}

\author{
Mozgovoy Vadym \\ Swiss Graduate School of Public Administration (IDHEAP) \\ University of Lausanne \\ Lausanne, Switzerland \\ vadym.mozgovoy@unil.ch
}

\begin{abstract}
Stress is a current issue in the workplace, manifesting itself through both psychological and physiological reactions. Biosensors might improve stress monitoring in the workplace, when employees become wearable device users. Yet, it remains unclear how to identify stress patterns through biosensors without direct observation of the users' activities. In particular, non-physiological aspects of employee activities altering physiological reactions, such as motion activity, may also be associated with stress measures. This longitudinal experimental study examines remote stress identification by testing whether a non-physiological signal of physical activity may improve the classification of stress-related physiological data collected through biosensors. The participants are 18 employees from Public Administration sector wearing biometric devices for around two months in the workplace. This study investigates the stress-related data classification, using established physiological measures (Galvanic Skin Response and Heart Rate) combined with a new non-physiological measure, associated with the user's physical activity (Motion Activity). Stress-related patterns are explored through unsupervised learning approach with help of Gaussian Mixture Model and K-Means classification analysis, completed by the bootstrap confidence intervals for evaluating uncertainty of classification. The results demonstrate that complementing physiological signals with a non-physiological signal, such as a physical activity-related information, improves stress pattern recognition through detection of emotional overarousal, arousal, and relaxation. These findings are especially promising in the context of the use of wearable devices for stress management, when stress-monitoring is done remotely and user' activity is not directly observed during measurements. Further research and cross-validation procedures should be used for building stress-identification algorithms for remote stress monitoring that include physiological and nonphysiological signals. Better understanding of stress measures may enhance the quality of stress management data collection processes through Information Systems, involved in the use of wearable devices in the workplace, and strengthen the data governance.
\end{abstract}

Keywords-data governance, stress, wearable biosensor, pattern recognition, classification

\section{INTRODUCTION}

Excessive stress in the workplace is an important problem for employees and organizations. Stress damages individual well-being of workers, decreases job performance and increases absenteeism. As a result, organizational expenses related to low job performance and sick leaves may rise. Furthermore, high blood pressure

Acknowledgment: This research has been supported by the Swiss National Science Foundation (SNSF) grant no. 172740.

The author thanks Prof. Ronchetti, during whose class "Selected Topics in Statistics" this project has been developed and presented in the Fall 2018. caused by stress among other reasons potentially undermines the heart health, which has been identified as the leading killer of Americans by American Heart Association [1].

According to the Encyclopedia of Behavioral Medicine [2], stress is defined as "a particular relationship between the person and the environment that is appraised by the person as taxing or exceeding his or her resources and endangering his or her well-being" [3, p. 19]. Stress may be caused either by mental stressors [3] or physical stressors [4] that individuals may encounter in private and professional activities. Events in external or internal environment and their perception represent mental stressors [3], while tasks of a physical nature, such as standing, pulling, manipulating objects, walking, or climbing stairs, represent physical stressors [4]. According to the mainstream classification of stress adopted by American Psychological Association (APA), stress may be acute, episodic acute, or chronic, whether it stems from mental or physical stressors in private life, workplace, or both [5, 6].

Acute stress is the most frequent and highly manageable stress form, representing isolated stress episodes. Moreover, experiencing an acute stress could be exciting, when it is associated with sports activities, for example. It stems from past, recent and anticipated demands and pressures, such as deadlines to meet, a presentation to make or a customer to serve in the workplace. It manifests itself through emotional distress, physical symptoms, and psychological signs of transient overarousal, such as dizziness, fast heartbeat, or high blood pressure. Acute stress makes a workplace a more thrilling place to be at, when its level is low or moderate.

Episodic acute stress represents frequently repeating acute stress episodes. It shares the symptoms with acute stress, but individuals experience them more frequently. Accordingly, this stress form becomes more difficult to manage and longer to treat. This stress form is more likely to stem from demands and pressures that individuals impose to themselves on frequent basis. Though individuals suffer from episodic acute stress, they may see it as a part of their lifestyle, which complicates its treatment. Episodic acute stress manifests itself through a variety of symptoms, such as chest pain, headaches, hypertension, and heart disease due to extended emotional and physical hyperarousal. The workplace becomes particularly stressful for individuals suffering from episodic acute stress, namely due to their irritability taken by other workers as real hostility.

Chronic stress is the most difficult form to manage and treat, because individuals get used to stress states, which may alter their personality. Contrary to acute stress, it does 
not excite or thrill individuals. It is associated with continuous demands and pressures that do not seem to end in an observable future. It usually has profound roots, such as a dysfunctional family or a traumatic childhood. The workplace experiences may also contribute to chronic stress, when individuals perceive that they are pursuing a despised career or are doing a deeply repugnant job. Chronic stress drains individual's physical and mental resources over long periods of time, and ultimately may lead to violence, heart stroke, and severe diseases, including depression and cancer.

The best way to counteract excessive stress is prevention. Remote stress monitoring involving stress data processing through Information Systems may contribute to preventing stress through its identification. Wearable biosensor technologies might possibly help monitoring and recognizing stress in the workplace. By workplace, this study understands the physical place or group of places where employees are located or move during working hours.

Though stress has three main forms, each of them inevitably manifests itself through psychological and physiological symptoms [2, 7, 8]. Accordingly, psychological and physiological measures are used to recognize stress independently or in combination. Though specific stress symptoms also vary from one individual to another [2], their presence facilitates stress detection and allows taking actions for counteracting stress before it becomes excessive.

Though literature distinguishes psychological and physiological measures of stress, wearable devices typically collect only the latter. At the same time, stress detection based on physiological signals is a complex process [5, 7]. In an aroused state typically signaling stress, a mental and physical activation occurs, the heart rate, sweating, and respiration accelerate, which is detected by the sensors in wearable devices. Yet, change incidence in physiological signals, such as blood pressure or cortisol levels, is not always alarming for individual wellbeing. For example, a professional sportsman's biometric device can reveal higher stress levels during training, which is a normal reaction to an intensive physical activity. Another example is an employee walking from one building to another during stress monitoring, which is completely safe for personal wellbeing. Related physiological pattern may be confounded with a stress instance in the context of remote monitoring.

Simultaneously, the physical activity is an important factor to consider in stress management, according to the theory of arousal explaining the links between arousal, performance, and stress. From a broader perspective, an arousal may not necessarily result in stress, because it is also related to other constructs, such as motivation and attention, and it is a fundamental concept in learning theories [9]. On one hand, an arousal preceding stress, which emerges when an individual in a normal state might prepare to a response to a potential stressor before returning to a normal state, has been described in the cognitive activation theory of stress [8].

On the other hand, hand the stress builds up as an inverted U-shaped function of the arousal level, according to the widely used Yerkes-Dodson law of arousal [10].
According to this law, the arousal level conceptualizes the capacity that an individual has at his disposition to perform tasks. Absent arousal or an arousal too low for an individual, representing a hypoarousal, will create inertia, boredom, dissatisfaction, and result in low performance. Thus, according to the Yerkes-Dodson law, the mid-level arousal allows optimizing the performance, pronging progress, change and fostering creativity. However, the arousal may go to a certain extent beyond the mid-point representing its moderate level for an individual performing a given cognitive or physical task. In this case, it will create a hyperactive effect, representing an overarousal, highly likely leading to stress.

A more recent Catastrophe Theory of arousal has extended this line of thinking and explained how the arousal beyond the moderate level affect an individual [11, 12]. Recent applications of this theory in other fields have confirmed this conceptualization $[12,13]$. It states that the physiological arousal associated with physical activity, such as sports, that goes beyond the mid-point might follow the inverted "U" pattern. However, it may occur under condition that the individual engaged in physical activity is not intensively anxious, nervous or worried.

By contrast, high level of such emotions, as intense anxiety representing an emotional overarousal, makes it more difficult to achieve the optimal moderate arousal point. Most importantly, an emotional overarousal in the case when an individual performs a demanding physical activity makes the performance decline rapidly and steeply shortly after the optimal performance has need attained. Finally, the recovery after this sharp performance decrease is very difficult, if possible at all.

Currently, there is a gap regarding physical activity in the methodological literature on stress pattern detection through data collected by wearable devices, connected to Information Systems. Stress identification procedures using biosensors need to be adapted to remote stress monitoring, when user activity cannot be directly observed. Such activities may include checking the work e-mails, participation in meeting, moving from one location to another during the working day, and so forth. This paper addresses this research problem in the workplace context.

Exploration of stress-related conditions is the purpose of this study. This study aims to explore how a nonphysiological signal indicating the users' motion intensity as a proxy of their physical activity may enhance the information on physiological signals in order to recognize stress-related patterns. A longitudinal experimental study was conducted to test a hypothesis on stress recognition, including motion activity. In this study, a non-physiological signal is represented by Motion Activity (MA). Physiological signals are measured through Galvanic Skin Response (GSR) and Heart Rate (HR). Performing stress type identification is outside the scope of this work. The following sections present the background on stress measurement for wearable devices, the research design, findings and conclusions.

\section{Stress Measures for WeArable DeVICES}

\section{A. Importance of Stress Measures}

Extensive research has been dedicated to stress identification involving psychological and physiological 
stress measures. Yet, individual non-physiological information related to the physical activity may contribute to revealing stress incidences. Thereby, it is important to complement physiological sensing results with additional information related to user's activity, in order to interpret correctly an observed stress occurrence.

\section{B. Physiological Measure of Stress}

This research considers physiological signals frequently used in stress identification problems and having strong relationship to stress - Galvanic Skin Response (GSR) and Heart Rate (HR). The GSR and HR are considered to be the biomarkers of stress, representing its autonomic physiological measures similarly to saliva while other type of physiological measures of hypothalamic-pituitaryadrenal activity are mainly based on cortisol [7]. The GSR and HR are widely used in psychological studies on arousal in the context of stress examination, learning, affect and other concepts, in which an arousal plays an important role [9]. GSR, HR and other physiological signals have been widely used in previous research for achieving two goals: 1) algorithm-based stress identification and 2) studies of stress-reactivity [14-16]. A combination of HR and GSR was shown to be a strong predictor of stress in a study on building fuzzy logic system for stress-detection [17]. Furthermore, it has outperformed alternative sets of predictors tested in that study in terms of cross-validation error.

HR reflects the number of heartbeats per unit of time [17]. In this study, it is measured in beats per minute "bpm". It is an established physiological measure of stress [7]. Empirical research evidence has demonstrated that HR may increase during periods of stress [17-19]. Previous research has also shown that the heart rate was affected during both acute and chronic stress through complex patterns [7].

GSR refers to electrodermal activity, measured in Kiloohm "kOhm" and associated to emotional arousal [20, 21]. When glands in the skin produce ionic sweat, they create alterations on electric conductivity, which is measured by GSR [17]. It has been previously used in stress detection research [22]. Specifically, emotional arousal may lead to increase in GSR.

Nevertheless, GSR and HR have limitations as arousal or stress measures. For instance, GSR or HR can increase due to increase of physical activity. In its turn, it leads to physiological arousal, which does not necessarily result in stress. Accordingly, there is a need to use indicators that allow alleviating this measurement issue.

\section{MOTION INTENSITY AS PROPOSED NON- PHYSIOLOGICAL MEASURE OF STRESS}

Physiological sensing reaches the limit of interpretability, no matter how high is prediction accuracy or measurement quality. However, combining physiological sensing with additional non-physiological variables characterizing user's activity can significantly improve stress identification procedures, as previous studies have shown [23].

Non-physiological signals are extensively used in extant research [7]. Yet, current studies do not account for the user's activity during remote stress monitoring in the workplace. At the same time, the use of non-physiological signals in the context of wearables in the workplace remains underexplored. The majority of currently used physiological measures of hypothalamic-pituitary-adrenal activity include chemical sensing of physical body reaction to stress [7]. The latter implies the delivery of glucocorticoid hormone cortisol from the adrenal cortex. Despite recent advances in cortisol sensing technologies, such instruments are beyond the scope of current study.

Current research proposes to address the research problem of remote stress pattern recognition by introducing a non-physiological signal - MA signal, characterizing the users' physical activity. It is hypothesized that the MA signal may improve the recognition of stress-related conditions in combination with HR and GSR. MA is a proposed non-physiological signal, tracking physical activity in a non-invasive way and allowing to monitor stress remotely without direct observation of the wearable device user. It is not produced by physiological or chemical processes of human being, and it is not a psychological stress signal neither.

This signal (MA) is operationalized as the intensity of user's motion. The High motion intensity will have positive relationship to HR, and may be a physical signal of stress. This newly proposed variable score ranges on a continuous scale from 0 to 100 . The following sections describes participants and methodology of the experiment conducted to test the hypothesized contribution that MA may have to stress recognition through wearables in the workplace.

\section{PARTICIPANTS}

The experimental study involved 18 participants from Municipal Fiscal Administration Office. Employees are Public Servants. The group included males and females of different age groups. They have been using wearable biometric devices in the period from 1 November 2018 to 18 December 2018. Most of the users (16 out of 18 ) have been wearing devices during working days. However, some of them ( 2 out of 18 ) used devices during the weekends as well.

\section{METHODS}

This section describes the research methods used in this work for stress pattern recognition. This work builds on experimental longitudinal research design. Wearable biometric devices (the wearables) with medical-grade measurement precision from specialized healthcare equipment manufacturer were used. The wearables recorded the users' signals during exploitation. The signals were typically registered with frequency 1 per second. Measurements that did not match the quality requirements of manufacturer were instantly detected and automatically deleted.

From the perspective of data analysis, this work assumes that stress-related conditions manifest themselves through latent variables or data clusters that we can identify. The following analytical steps are taken: 1. Perform data exploration; 2. Identify stress-related conditions; 3 . Suggest stress detection procedures for future research and validation. Statistical software involved in this work is MATLAB and R.

Data analysis relies on closely connected algorithms: KMeans and Expectation-maximization (EM) algorithm for Gaussian Mixture Model (GMM). K-means algorithm represent a special non-probabilistic limit of EM applied to 
mixtures of Gaussians [24]. Both models are suitable for clustering. However, the GMM assumes a Gaussian form for Mixture components considering that latent variables define the attribution of data points to specific components of the mixture. In addition, the GMM is frequently used in pattern recognition, data mining, machine learning and statistical analysis [24].

Furthermore, the GMM model and K-Means algorithm are techniques used for recognizing stress-related patterns. They suit for this problem particularly well. It is assumed that stress-related conditions express themselves by forming distinctive groups, that can be observed through a combination of physiological measure (GSR and HR) and non-physiological measure (MA), representing a physical activity signal. Past research on real-time stress detection by means of physiological signal have already applied GMM and K-Means classifiers, though it was done in the context of supervised learning problem [17].

In this study, data exploration is performed through the use of K-means and GMM. K-means technique is applied for general non-probabilistic classification without assuming any data structure. GMM refines the analysis identifying the latent variables related to stress assuming normality of the data. In addition, GMM allows adopting probabilistic perspective of cluster assignment (soft clustering) and evaluating uncertainty with help of bootstrap confidence intervals. Further, we analyze the similarity of classification at 10 different initiations of algorithms in order to check the robustness of K-Means and GMM classification.

Both methods (K-Means and GMM) facilitate identification of stress-related conditions. They complement each other allowing for both probabilistic and nonprobabilistic classification analysis. Finally, they are used for stress pattern recognition and may help to establish stress identification procedures for future research and validation.

\section{A. K-means Algorithm}

The main idea of algorithm is to minimize the objective function (1). In other words, we assign $\mathrm{n}^{\wedge}$ th data point to the closest data center. It corresponds to hard clustering. The KMeans technique is attractive for starting an exploration, because no distributional assumptions should be held.

$$
J=\sum_{n=1}^{N} \sum_{k=1}^{K} r_{n k}\left\|x_{n}-\mu_{k}\right\|^{2}
$$

In (1) for each data point $x_{n}(\mathrm{n}=1 \ldots \mathrm{N})$, we define binary variables $r_{n k} \in\{0,1\}$, where $\mathrm{k}=1, \ldots, \mathrm{K}$ represents a cluster to which the data point $x_{n}$ is assigned to. If $x_{n}$ is assigned to cluster $\mathrm{k}$, then $r_{n k}=1$, correspondingly $r_{n j}=0$ for $\mathrm{j} \neq \mathrm{k}$. See [24] for more details.

\section{B. Gaussian Mixture Model}

In the GMM, we aim to maximize a likelihood function (1) using an EM algorithm. As a result, we obtain a probability with which an observation $\mathrm{x}$ belongs to a component $\mathrm{k}$. It corresponds to soft clustering and requires a parametric assumption to be held, stating that mixture components are normally distributed. The GMM is attractive, because stress-related patterns may be considered as latent variables manifested through physiological signals.

$$
\ln p(X \mid \pi, \mu, \Sigma)=\sum_{n=1}^{N} \ln \left\{\sum_{k=1}^{K} \pi_{k} \mathcal{N}\left(x_{n} \mid \mu_{k}, \Sigma_{k}\right)\right\}
$$

In (2), $p\left(z_{k}=1\right)=\pi_{k}$ corresponds to prior probability and $p\left(x \mid z_{k}=1\right)=\mathcal{N}\left(x \mid \mu_{k}, \Sigma_{k}\right)$ corresponds to Gaussian distribution; $x_{n}(\mathrm{n}=1 \ldots \mathrm{N})$ represents data point and $\mathrm{k}=$ $1, \ldots, K$ represents a cluster index. See [24] for more details.

\section{VI.PRE-PROCESSING AND FEATURE EXTRACTION}

Sampling procedure consists in randomly selecting 3 days of exploitation for each of 18 users. Overall, 14 working days of November and 10 working days from December appear in the sample comprising of 1'018'497 observations (i.e. around 283 hours of device use in total). Each observation corresponds to the signal value for particular second. Next, sliding windows technique with step size of 60 seconds without overlap has been used. At each step, an average value of selected observations has been computed. Finally, after removing missing values and creating averages dataset comprised 14'133 features. Each feature consisted of interval averages of GSR, HR and MA.

\section{RESULTS}

\section{A. K-Means Classification Analysis}

Stress-related conditions may manifest themselves through identifiable groups of similar physiological responses. Thus, we consider three assumptions for justifying the choice of variables and specified number of clusters:

1) Emotional overarousal state may be correlated to high degree of emotional arousal and elevated HR. It is highly likely to indicate that an individual is experiencing a stress episode now or will be likely to do so in the near future.

2) Arousal state may be correlated to low degree of emotional arousal, high motion intensity and elevated HR. This cluster is associated with the process of completing a physically demanding task, which may possibly lead to an acute stress episode $[6,25]$. However, it will not necessarily occur. For this reason, it is labeled as "arousal state" for analytical purposes.

3) Phase of relaxation may be related to low degree of emotional arousal and low motion intensity.

We select three variables (GSR, HR and MA), where HR expresses the heart rate, GSR expresses degree of emotional arousal and MA corresponds to motion intensity. We add a non-physiological signal MA into classifier to account for physical activity that may increase the levels of GSR and HR. If an increase in GSR or HR is associated with increase of physical activity, then increase of GSR or HR will correspond to the increase of Motion Intensity. Correspondingly, a scatterplot will reveal a region where increase of GSR or HR is followed by increase of MA and classifier may identify it and form corresponding cluster.

On another hand, we aim to identify clusters having relationship to (1) Emotional over-arousal state; (2) Arousal state; (3) Phase of Relaxation. Thus, we run K-Means algorithm on GSR; HR and MA specifying 3 clusters without any distributional assumption. In other words, $\mathrm{k}=3$ refers to an assumption that there exist three distinguishable states. 
Finally, we obtain classification visualized on Fig. 3 comprising of Blue Cluster; Red Cluster and Green Cluster. As the Figure below shows, conditions related to the emotional overarousal are observed less frequently, compared to other conditions. Thus, the relative weight of Red Cluster is $10 \%$ of cases, whereas the weight of Green Cluster is $17 \%$ and Blue Cluster $72 \%$ respectively.

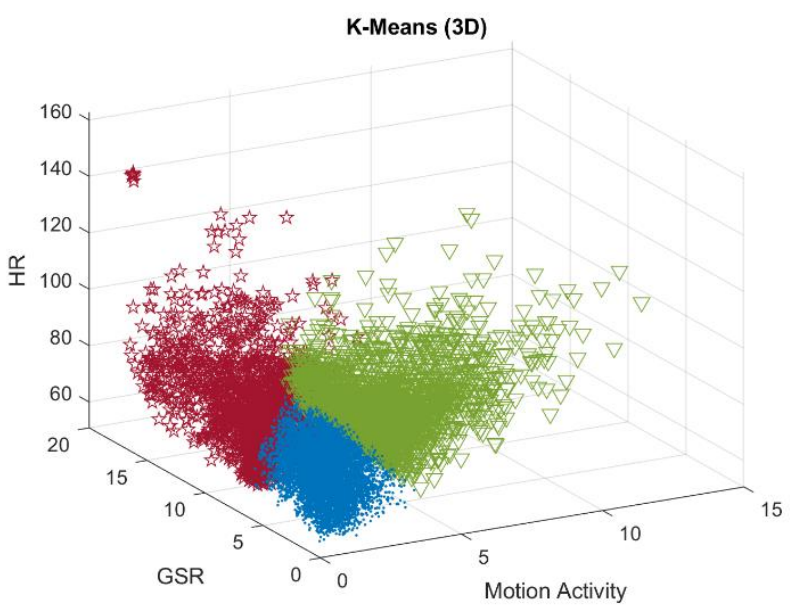

Fig. 3. K-Means scatter plot with 3 clusters in 3D

Blue Cluster lies in the lowest range of variables GSR, MA and HR among all the clusters. Averages of those variables corresponding to Blue Cluster are smaller than averages for all other clusters. For example, average GSR (2.92) for Blue Cluster is less then corresponding GSR average for red cluster (10.39) and Green Cluster (3.18) (see Table 1). It represents the lowest degree of emotional arousal, motion intensity and heart rate then other groups. Therefore, this cluster may represent the physiological response to the state of Relaxation.

TABLE I. Cluster AVERAGES

\begin{tabular}{|l|l|l|l|}
\hline \multirow{2}{*}{ Signal } & \multicolumn{3}{|c|}{ Cluster } \\
\cline { 2 - 4 } & \multicolumn{1}{|c|}{ Blue } & \multicolumn{1}{c|}{ Red } & \multicolumn{1}{c|}{ Green } \\
\hline GSR & 2.9216 & 10.3925 & 3.1849 \\
\hline HR & 72.2196 & 78.7131 & 91.4036 \\
\hline MA & 1.3276 & 1.8897 & 3.4544 \\
\hline
\end{tabular}

Green Cluster lies in the low range of GSR. For example, average GSR for this cluster (3.18) is nearly the same as GSR of Blue Cluster (2.92), but it is much smaller than GSR for red cluster (10.39). So, this group is not associated with elevated degree of emotional arousal in comparison to other groups. On another hand, a dependency is observed between MA and HR inside cluster. Fig. 4 illustrates that an increase in MA is followed by increase in HR. Finally, Green Cluster (characterized by elevated motion intensity, elevated HR and low emotional arousal in comparison to other groups) demonstrates a stronger connection to an arousal state.

Red Cluster corresponds to the highest GSR interval relatively to other clusters. Accordingly, average cluster GSR is of 10.3925, which is the highest grade among other groups. At the same time, the average HR (78.71) is higher than in Blue Cluster (72.22), but lower than in Green Cluster
(91.40). This group is characterized by elevated emotional arousal, elevated HR and low motion intensity. It has stronger connection to stress episodes than other groups. However, Red Cluster may be also connected to other emotional reactions such as anxiety, or strong "positive stress".

Finally, we deduce the following insights from the KMeans classification:

1) Blue Cluster may be related to a state of relaxation;

2) Green Cluster may be related a state of performing a demanding physical activity, indicating an arousal state;

3) Red Cluster may be related to an emotional overarousal state.

The robustness of K-Means classification was analyzed to evaluate the similarity of classification. At first, we run the algorithm 10 times (from seed 1 to seed 10). Then, we arrange obtained cluster centroids into three groups: Blue, Red and Green. The Blue group has the lowest MA, the lowest HR, and the lowest GSR among all the groups. The Red group has the highest GSR among all the groups and elevated HR. The Green group has the highest MA among all the groups and elevated HR.

This classification does in line with the cluster centroids arranged in three groups in Fig.3 and its corresponding description. As a result, we have successfully distinguished between 3 groups characterized by links to mental stress, overarousal, and relaxation 9 times out of 10 . Next, we computed medians of each cluster sentoids across 9 seeds shown on Table 2 . They are remarkably close to the output from Table 1, confirming the reported results of K-Means classification.

TABLE II. CLUSTER MEDIANS FOR 9 SEEDS

\begin{tabular}{|l|l|l|l|}
\hline \multirow{2}{*}{ Signal } & \multicolumn{3}{|c|}{ Cluster } \\
\cline { 2 - 4 } & \multicolumn{1}{|c|}{ Blue } & \multicolumn{1}{c|}{ Red } & \multicolumn{1}{c|}{ Green } \\
\hline GSR & 2.9298 & 10.4684 & 3.1849 \\
\hline HR & 72.1889 & 78.7131 & 91.4036 \\
\hline MA & 1.3259 & 1.894 & 3.4544 \\
\hline
\end{tabular}

However, 1 time out of 10 , i.e. in seed 8 , classification output did not match a pattern described above. One of the clusters had highest GSR and highest MA and the same time. It means that classification failed to distinguish between conditions linked to arousal and emotional overarousal and it fused Red and Green Groups in one single group.

Therefore, finally reported classification outputs are rather stable over different algorithm initiations and in general they lead to same three distinguishable states. Hence, data suggests a stable pattern observed over most of the seeds. Thus, repeated classification over multiple seeds and performed visual analysis of the clusters support the selection of the most frequent result. 


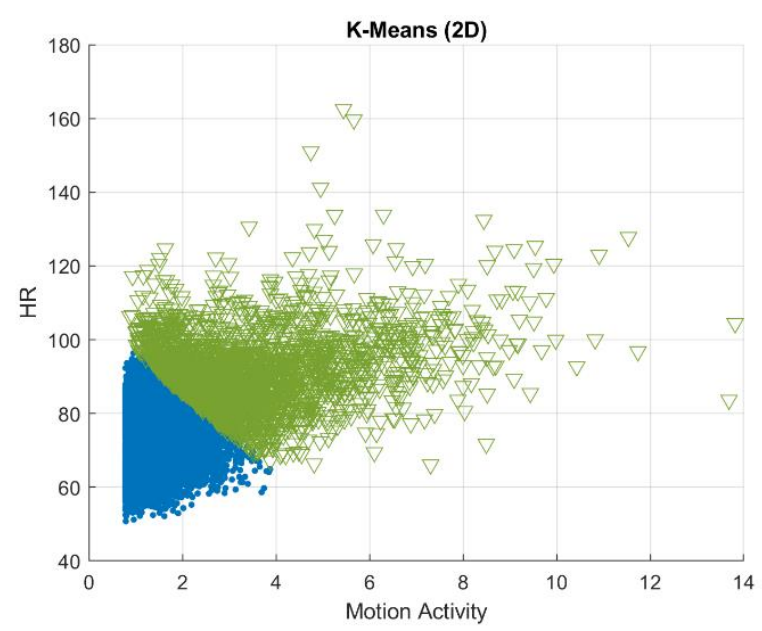

Fig. 4. K-Means scatter plot with 2 clusters in 2D

Our analysis goes further, and we apply an alternative model to interpret the empirical data. Classification algorithm, distributional assumptions, algorithm initiations (such as number of clusters considered), initial dataset may influence the results. Thus, we consider an alternative Model for classification analysis - GMM.

In order to refine the analysis, we focus on conditions related to emotional overarousal. For this purpose, we create a new dataset by excluding observations related to an arousal state (i.e. Green Cluster from K-Means classifier) and reducing dimensionality. It aims to reduce the impact of physical motion on stress-related physiological responses. Thus, we use this restricted dataset in GMM analysis. In this case, we will focus on the most prominent available biomarkers for stress-related conditions: GSR and HR.

\section{B. GMM Classification Analysis}

Conditions related to emotional overarousal can be viewed as latent variables expressed by data patterns. GMM analysis helps to investigate patterns related to emotional overarousal and possibly identifying the stress episodes. Therefore, we specify a finite mixture model consisting of 3 clusters. We assume that we can identify several different, but potentially overlapping states. We specify 3 clusters considering existence of low, medium and high arousal ranges. A contour plot of fitted model and scatter plot are represented on Fig. 5.

Left Cluster on the Fig. 4 has lower range of emotional arousal and HR (60-100 bpm), then the other groups. The relative weight of cluster is around $67 \%$. This is the most common state in the workplace, and may be related to the state of relaxation.

Central cluster has middle range of emotional arousal and same HR range as in left cluster. The relative weight of cluster is around $24 \%$. It is the second most common state in the workplace, and can reflect normal working routine. Moderate stress is not damaging for personal wellbeing, allows achieving optimal performance levels, and has positive effects of bringing excitement and thrill to the workplace [6].

Right cluster on the Fig.5 has the highest range of emotional arousal and elevated heart rate range, compared to other clusters. There were several outlying observations with very high HR (around 150 "bpm") and elevated GSR (around 18 "kOhm") at the same time. Right cluster is less common in the workplace. It contains only around $8 \%$ of observations. Thus, it may be related to emotional overarousal states and other conditions associated with high emotional arousal.

Further GMM classification was performed 10 times for different seeds on a subset of data described earlier in order to analyze the robustness. Multiple repetitions yielded exactly the same result.

The Fig.5 shows overlapping classes of observations. From probabilistic perspective, one observation will have a chance to belong to several clusters. There is no clear separation between the clusters. The distinction between different states is thus fuzzy justifying soft clustering approach. At the same time, the attribution probability will increase when observations lie closer to center of some component.

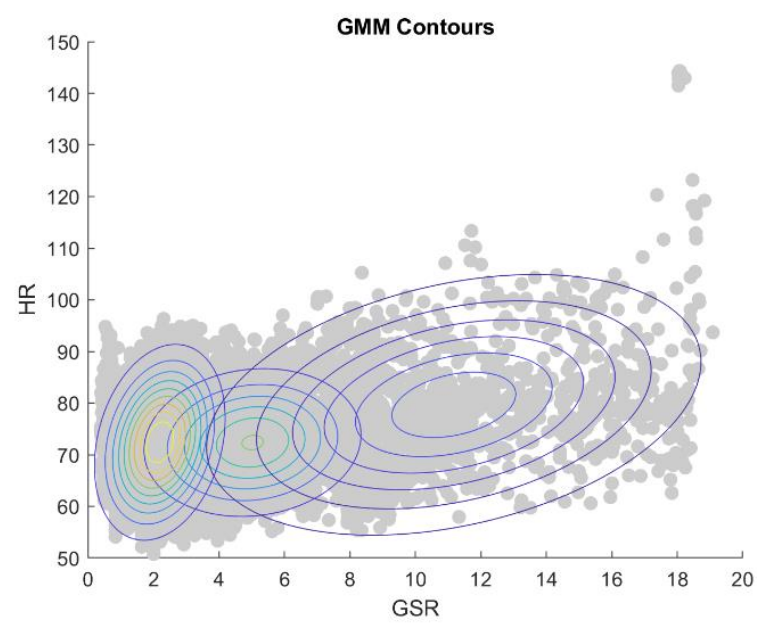

Fig. 5. GMM Contours and Scatter Plot

Weighted likelihood bootstrap confidence Intervals are narrow and show high degree of certainty in parameter $\mu$ for left and central clusters (see Table 2). Although the uncertainty is higher for Right Cluster (compared to other groups) the size of intervals is relatively low with respect to the scale of GSR and HR. Overall, the confidence in point estimates of cluster centers is high.

TABLE III. WEIGHTED LIKELIHOOD BOOTSTRAP

\begin{tabular}{|l|c|c|}
\hline \multirow{2}{*}{$\begin{array}{c}\text { Confidence Interval } \\
\text { for } \boldsymbol{\mu}\end{array}$} & $\boldsymbol{G S R}$ & $\boldsymbol{H} \boldsymbol{R}$ \\
\cline { 2 - 3 } & 2.180413 & 72.02012 \\
\hline Left cluster $2.5 \%$ & 2.282331 & 72.65056 \\
\hline Left cluster $97.5 \%$ & 4.977984 & 72.01403 \\
\hline Central cluster $2.5 \%$ & 5.398048 & 72.95102 \\
\hline Central cluster $97.5 \%$ & 9.831141 & 76.89023 \\
\hline Right cluster $2.5 \%$ & 11.853478 & 80.99914 \\
\hline Right cluster $97.5 \%$ & & \\
\hline
\end{tabular}




\section{DISCUSSION}

\section{A. Findings}

This research has examined whether motion intensity might be a non-physiological indicator contributing to stress monitoring in the context of wearable device use. This experimental study has found that combining physiological signals reactive to stress (HR and GSR) with nonphysiological signal (MA) reflecting physical activity allows to recognize patterns related to stress in the workplace. This study identifies three responses that may be related to (1) emotional overarousal state, (2) arousal state, (3) state of relaxation, and corresponding similarity criteria. They are represented by cluster centroids in case of $\mathrm{K}$ Means and distributional parameters in case of GMM.

Despite vast existing research on stress detection, extant procedures are not always adopted to remote stress monitoring. Most of existing studies assume knowing activity of user for identifying stress-related information and interpreting it. This study shows that combining two types of signals from biometrical sensing device can contribute to development of remote stress monitoring procedures including stress detection, when activity of user is not directly observed. It also suggests two alternative approaches that can be further examined in the context of remote stress detection. Both of them incorporate MA, GSR and HR. The first one is based on K-Means (procedure 1). The second assumes taking out observation related to the intensive motion and implement GMM on GSR and HR couple (procedure 2).

The result of this study go in line with the psychology literature on arousal and stress. According to the Catastrophe theory of arousal [11, 12], the physical activity may not necessarily result in stress. However, physiological arousal associated with high levels of emotions, such as anxiety, are likely to impede the achievement of an optimal moderate arousal point, and may provoke stress very shortly after it has been reached. Furthermore, a combination between an emotional overarousal and physical activity might quickly result in stress.

Three categories of physiological arousal identified in this study could be interpreted from different perspectives. The use of category analytically labeled as "relaxation state" allows identifying normal state of the user. However, this category could be relevant in the context of stress prevention, because it signals when user's physiological arousal level is below the mid-point. If this state persists over time, it suggests that an individual is in a hypoarousal or is insufficiently challenged, which might lead to stress in the future.

The use of category analytically labeled as "an emotional overarousal state" could be relevant in the context of stress management, because it signals when stress episodes are the most likely to occur. Emotional overarousal state is defined as increased emotional arousal state in the literature on stress [6]. If stress instances are detected, then appropriate coping strategies could be adopted, too [2]. Yet, depending on individual physiological reactions, an overarousal may not necessarily be an indicator of stress. In parallel, stress may manifest itself through a range of symptoms that may vary from one individual to another. Thus, if an individual is not overaroused, it may not necessarily indicate the absence of stress. Furthermore, an emotional overarousal might also a symptom of other states, such as hyperarousal or a wider range of mental health disorders outside of the scope of this study [26].

In parallel, it should be noted that the cluster combining high levels of emotional arousal during high level of motion was not identified during the experiment. It shows that there was no recurrent pattern of acute stress incidence associated with demanding physical activity. However, this pattern could emerge in other workplaces that have other characteristics.

The use of category analytically labeled as "an arousal state" could be particularly relevant in the context of stress prevention, because it signals a potential stress condition before it actually occurs. An arousal allowing to produce and prepare a response to a potentially stressing event has been described in the literature on stress [8]. Though it may be unpleasant at times, it is not harmful on its own, and the physiological signals return back to normal after the challenge has been successfully addressed.

Completing a physically demanding task may not necessarily lead to an acute stress episode, especially if it is not recurrent or when an individual has sufficient physical resources to meet physical demands associated with this task. Physically demanding tasks may be completed without negative effects on personal well-being.

Yet, a completion of physically demanding task may possibly result in an acute stress [6]. In this context, a low degree of emotional arousal, high motion intensity and elevated HR can also be seen as an effort preceding the stress episode. If frequently repeated and going beyond physical resources of an employee, the physical activity may result in an acute episodic stress. If physical demands are continuous, overwhelming and there is no perspective of change, an individual may develop chronic stress. Accordingly, the data on motion should be interpreted in the context of job design and activity sector of an organization.

These findings confirm that algorithms and analytical procedures based uniquely on physiological signals are limited in distinguishing between stress episodes and physiological arousal level only potentially leading to stress. There is a need to treat signals differently in order to make correct insights from the stress-related indicators. For example, an anomaly detection may require different analytical approach in case when the user performs physically demanding activities. It is especially relevant in the context of wearable devices when users move a lot. Current research demonstrates that MA variable indirectly characterizing human activity has large potential to improve algorithms and procedures for remote stress monitoring, as well as contribute to stress prevention in the workplace.

\section{B. Limitations}

This study is subject to limitations. It is important to highlight that this work is not performing stress type identification, but only explores stress-related conditions. For example, stress outside of the work could hardly be separated from work stress in terms of physiological response that a body produces to it. People often bring home stress to the work and work stress to home. Thus it may be possible to develop special monitoring programs where users wear the devices outside of the work environment under their consent. 
Next, current results may be influenced by using different classification algorithm, stress-detection procedure or initial sample. However, in order to support the relevance of GSR, HR and MA in stress pattern recognition we have applied two alternative classification schemes. Both of them confirm that we can extract meaningful insights from the data. Moreover, a large dataset consisting of around 283 hours of use has been considered in order to support the credibility of findings.

This study concerns observations mainly taken during working hours in the office setting. Physiological signals and non-physiological signals, such as physical signals taking form of motion intensity measures, may vary. Their character and variability in different work environment may differ substantially and follow distinctive stress-related patterns.

The use of different biomarkers indicating physiological reactions will also have an impact on results stress recognition. For example, sensing cortisol, which is beyond the scope of current work, may strongly influence remote stress pattern recognition. However, chemical sensing is not currently widespread in wearable devices, in particular, due to ergonomic considerations.

One of the study limitations is the lack of psychological measures allowing to combine the data and improve stress detection. However, our research problem is centered on the remote stress detection through wearables. Currently, remote stress detection does not imply the use of such measures.

Another limitation is the interpretation of similarity groups. Additional empirical observations are needed in order to confirm current data interpretation. Data collection on individual performance levels that could help interpreting the arousal levels was outside of the project scope. Furthermore, clusters represent multiple conditions. For example, cluster related to emotional overarousal states will correspond to high degree of emotional arousal, thus including conditions of anxiety and "positive stress" as well. Thus, the interpretability of identified groups has limitations. The refined interpretation will demand additional sources of information.

\section{Implications}

This study has several implications. First of all, it may contribute to development of meaningful and interpretable stress-related indicators for remote stress monitoring. Such indicators should be based on validated and adopted stressdetection procedures for remote stress monitoring. At second this study may contribute to the development of new stress-monitoring practices, processes and procedures in the workplace.

\section{Future Research}

In the future research on remote stress detection, other approaches to incorporating MA or other non-physiological variables into stress-pattern recognition should be examined further. Future studies should also consider examining stress identification procedures, adapted to three different acute, acute episodic and chronic stress types [5, 25], because their symptoms follow different patterns. Furthermore, different stress types send different physiological signals [8]. In this context, a multi-method approach combining the physiological, physiological and physical measures could be particularly relevant.

Future research should also consider cross-validation of procedures used in this work in the context of remote stress identification in the workplace. It may involve alternative validated instrument for stress identification, such as questionnaires. Stress incidence identified with help of current procedures should demonstrate significant correlation to alternative and validated instruments for stress identification in order to confirm their relevance for remote stress monitoring. Otherwise, stress induction procedure could be considered for proving validity of suggested approach. However, it would demand a clinical trial for data collection which is expensive.

Future research on stress pattern recognition can be done in order to analyze the strains of specific workplaces and design corresponding preventive actions. At the same time, it may allow to compare patterns from one working environment to another by comparing the relative weight of different clusters and their respective characteristics.

Finally, research can be done in order to establish zones of safety and risk for long-term remote stress monitoring. Risk zone will correspond to the overarousal observed during certain interval of time with help of device, especially during high motion activity, which may indicate excessive stress-related incidence. This average incidence may be connected to combination of sick leaves, absenteeism and decrease of work-related performance and possibly can predict harmful stress-related states. Identification of this link may help to define a mid-point threshold of physiological arousal, when excessive stressrelated incidence becomes potentially damaging for individual well-being. Intervals of risk may serve as guidelines for stress-management in the workplace. For example, occupational health specialist may observe stressrelated indicators, reaching the risk zone on the departmental level in the workplace. Consequently, psychosocial interventions for preventing and managing stress may be effectively designed in order to improve wellbeing of employees.

\section{CONCLUSION}

This study advances the understanding of stress-related patterns identified through wearable biometric devices in the workplace by examining how non-physiological signals may contribute to stress pattern recognition. The longitudinal experiment in Swiss Public Service conducted with 18 participants over two-months period has shown that motion intensity is a non-physiological signal improving stress pattern recognition in combination with physiological signals.

These results may contribute to stress monitoring in the context of the use of wearable devices in the workplace. By extension, better understanding of stress measures may strengthen the data governance process related to the stressmanagement in the workplace. Further research is needed to validate these exploratory findings and building robust stress-identification algorithms for remote stress monitoring that includes physiological and non-physiological signals.

\section{REFERENCES}

[1] American Heart Association, "Stress and Heart Health," American Heart Association, Apr. 2018. [Online] Available: 
https://www.heart.org/en/healthy-living/healthy-lifestyle/stressmanagement/stress-and-heart-health\#.WrlAbdTwaUk. Accessed on: Apr. 282019.

[2] S. Folkman, "Stress: Appraisal and Coping," in Encyclopedia of Behavioral Medicine, M. D. Gellman and J. R. Turner, Eds., New York, NY: Springer New York, 2013, pp. 1913-1915.

[3] R. S. Lazarus and S. Folkman, Stress, appraisal and coping. New York: Springer Publishing Company, 1991.

[4] G. S. Anderson, R. Litzenberger, and D. Plecas, "Physical evidence of police officer stress," Policing, vol. 25, no. 2, pp. 399 420, 2002

[5] L. H. Miller, A. D. Smith, and L. Rothstein, The stress solution: An action plan to manage the stress in your life. New York: Pocket Books, 1994.

[6] American Psychological Association, "Stress: The different kinds of stress," American Psychological Association (APA), 2019. [Online] Available: https://www.apa.org/helpcenter/stress-kinds. Accessed on: Apr. 282019.

[7] S. J. Lupien, "How to Measure Stress," Center for Studies on Human Stress, Louis-H. Lafontaine Hospital, 2013. [Online] Available: http://www.stresshumain.ca/Documents/pdf/Mesuresphysiologiques/CESH_howMesureStress-MB.pdf. Accessed on: Apr. 282019

[8] H. Ursin and H. R. Eriksen, "The cognitive activation theory of stress," Psychoneuroendocrinology, vol. 29, no. 5, pp. 567-592, 2004.

[9] W. Revelle and D. A. Loftus, "The implications of arousal effects for the study of affect and memory," in The handbook of emotion and memory, S.-Å. Christianson, Ed., Hillsdale N.J.: L. Erlbaum Associates, 1992, pp. 113-149.

[10] R. M. Yerkes and J. D. Dodson, "The relation of strength of stimulus to rapidity of habit-formation," J. Comp. Neurol. Psychol., vol. 18, no. 5, pp. 459-482, 1908.

[11] J. Fazey and L. Hardy, The inverted-U hypothesis: catastrophe for sport psychology., 1988

[12] L. Hardy and G. Parfitt, "A catastrophe model of anxiety and performance," (eng), British journal of psychology (London, England : 1953), vol. 82 ( Pt 2), pp. 163-178, 1991.

[13] P. Vitasari et al., "Assessing of Physiological Arousal and Cognitive Anxiety toward Academic Performance: The Application of Catastrophe Model," Procedia - Social and Behavioral Sciences, vol. 30, pp. 615-619, 2011.

[14] J. Healey and R. Picard, "SmartCar: Detecting driver stress," in Proceedings 15th International Conference on Pattern Recognition ICPR-2000, 2000, pp. 218-221.

[15] K. Palanisamy, M. Murugappan, and S. Yaacob, "Multiple physiological signal-based human stress identification using non- linear classifiers," Elektronika ir elektrotechnika, vol. 19, no. 7, pp. 80-85, 2013.

[16] J. Zhai and A. Barreto, "Stress detection in computer users through non-invasive monitoring of physiological signals," Blood, vol. 5, no. 0, 2008.

[17] A. de Santos, C. Sánchez-Avila, J. Guerra-Casanova, and G. Bailador-Del Pozo, "Real-time stress detection by means of physiological signals," in Recent Application in Biometrics: IntechOpen, 2011.

[18] A. de Santos Sierra, C. S. Avila, J. G. Casanova, G. B. del Pozo, and V. J. Vera, "Two stress detection schemes based on physiological signals for real-time applications," in Intelligent Information Hiding and Multimedia Signal Processing (IIH-MSP), 2010 Sixth International Conference on, 2010, pp. 364-367.

[19] M. A. Birkett, "The Trier Social Stress Test protocol for inducing psychological stress," Journal of visualized experiments: JoVE, no. 56, 2011.

[20] J. Zhai, A. B. Barreto, C. Chin, and C. Li, "Realization of stress detection using psychophysiological signals for improvement of human-computer interactions," in Proceedings. IEEE SoutheastCon, 2005, 2005, pp. 415-420.

[21] F. Angus, J. Zhai, and A. Barreto, "Front-end analog preprocessing for real-time psychophysiological stress measurements," in Proceedings of the 9th World Multi-Conference on Systemics, Cybernetics and Informatics (WMSCI 05), 2005, pp. 218-221.

[22] M. N. Taib, Ed., IEEE 7th International Colloquium on Signal Processing and Its Applications (CSPA), 2011: 4 - 6 March 2011, Penang, Malaysia ; proceedings. Piscataway, NJ: IEEE, 2011.

[23] E. Garcia-Ceja, V. Osmani, and O. Mayora, "Automatic Stress Detection in Working Environments from Smartphones' Accelerometer Data: A First Step," IEEE J. Biomed. Health Inform., vol. 20, no. 4, pp. 1053-1060, http://arxiv.org/pdf/1510.04221v1, 2016

[24] C. M. Bishop, Pattern Recognition and Machine Learning: Springer New York, 2016

[25] M. R. Frone, M. Russell, and M. L. Cooper, "Antecedents and Outcomes of Work-Family Conflict: Testing a Model of the Work -- Family Interface,” The Journal of applied psychology, vol. 77, no. 1, pp. 65-78, 1992.

[26] E. Haralanova, S. Haralanov, A. Beraldi, H.-J. Möller, and K. Hennig-Fast, "Subjective emotional over-arousal to neutral social scenes in paranoid schizophrenia," (eng), European archives of psychiatry and clinical neuroscience, vol. 262 , no. 1 , pp. $59-68$, 2012 